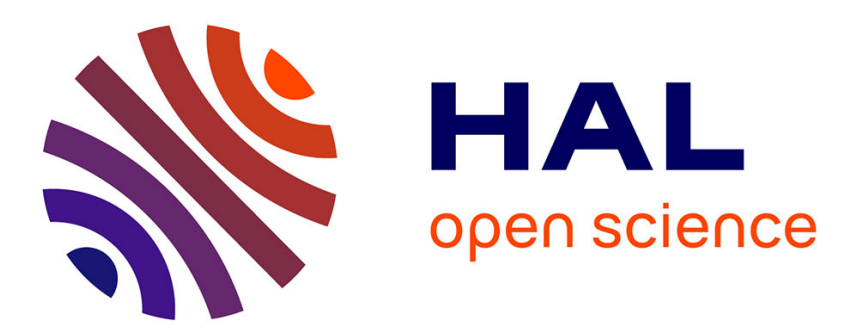

\title{
Air bubbles under vertical vibrations
}

Farzam Zoueshtiagh, H. Caps, M. Legendre, N. Vandewalle, P. Petitjeans, P

Kurowski

\section{To cite this version:}

Farzam Zoueshtiagh, H. Caps, M. Legendre, N. Vandewalle, P. Petitjeans, et al.. Air bubbles under vertical vibrations. European Physical Journal E: Soft matter and biological physics, 2006, 20 (3), pp.317 - 325. 10.1140/epje/i2005-10131-6 . hal-02453945

\section{HAL Id: hal-02453945 \\ https://hal.science/hal-02453945}

Submitted on 24 Jan 2020

HAL is a multi-disciplinary open access archive for the deposit and dissemination of scientific research documents, whether they are published or not. The documents may come from teaching and research institutions in France or abroad, or from public or private research centers.
L'archive ouverte pluridisciplinaire HAL, est destinée au dépôt et à la diffusion de documents scientifiques de niveau recherche, publiés ou non, émanant des établissements d'enseignement et de recherche français ou étrangers, des laboratoires publics ou privés. 


\title{
Air bubbles under vertical vibrations
}

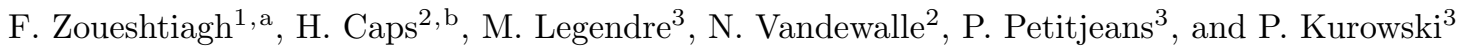 \\ 1 LML, Université de Lille 1, UMR 8107 CNRS, Bd Paul Langevin, F-59655 Villeneuve d'Ascq, France \\ 2 GRASP - Université de Liège, Institut de Physique B5, B-4000 Liège, Belgium \\ 3 PMMH - École Supérieure de Physique et Chimie Industrielles (ESPCI), UMR 7636 CNRS, 10 rue Vauquelin, F-75231 Paris \\ Cedex 05, France
}

Received 14 November 2005 and Received in final form 30 May 2006 /

Published online: 28 July 2006 - (c) EDP Sciences / Società Italiana di Fisica / Springer-Verlag 2006

\begin{abstract}
This paper reports on an experimental study of the splitting instability of an air bubble a few centimetres in diameter placed in a sealed cylindrical cell filled with liquid and submitted to vertical oscillations. The response of the bubble to the oscillations is observed with a high-speed video camera. It is found that the bubble dynamics is closely associated with the acceleration of the cell $\Gamma$. For small acceleration values, the bubble undergoes minor shape deformations. With increasing acceleration values, these deformations are amplified and for sufficiently large $\Gamma$ the bubble becomes toroidal. The bubble may then become unstable and split into smaller parts. The onset of bubble division is studied and its dependency on physical parameters such as the fluid viscosity, the fluid surface tension and the initial size of the bubble is presented. It is found that the criterion for the bubble splitting process is associated with a threshold based on the acceleration of the oscillations. Above this threshold, the number of bubbles present in the cell is observed to grow until a final steady state is reached. Data analysis reveals that the final bubble size may be characterized in terms of Bond number.
\end{abstract}

PACS. 47.55.dd Bubble dynamics - 47.35.Pq Capillary waves - 47.20.Dr Surface-tension-driven instability

\section{Introduction}

Bubbles can be easily formed by mixing two fluid jets or blowing a gas into a liquid phase [1-3]. The common presence of bubbles in our everyday life makes them play an important role in a wide range of geophysical and industrial processes [4-6]. For instance, the performance of an ordinary water-pump can be directly affected by the presence of air bubbles in its water circuit. Therefore, the presence of air bubbles is widely taken into account during the early stages of hydraulic design.

Since Faraday reported on the effects of forced oscillations of a liquid layer [7], numerous studies have focused on the various aspects of vibrations on liquid layers, films or drops (see, e.g., Refs. [8-14]). These studies have led to a better understanding of fluid behaviour and, consequently, have paved the way for new industrial processes. For instance, James et al. [11] recently reported on a new method for atomizing a liquid drop by bursting it using a vibrating diaphragm. Another example is the work accomplished by Wilkes et al. [13] who brought forward the use of an oscillating rod on a drop-on-demand technology with promising applications in microfluidics.

\footnotetext{
a e-mail: Farzam.Zoueshtiagh@univ-lille1.fr

b e-mail: herve.caps@ulg.ac.be
}

Similarly, the forced oscillation of a bubble has also received attention. Many experimental and theoretical works have been conducted on small (radius $\leq \mathrm{mm}$ ) bubbles with various practical applications (see, e.g., [15-19]). However, it appears that the stability of a large bubble (radius of the order of $\mathrm{cm}$ ) has attracted less attention. To our knowledge the few reported works are those of references $[17,18]$ where the induced motion of a single bubble arising from vertical vibrations was studied in microgravity.

In this paper, a large air bubble is set inside a sealed cylindrical cell filled with liquid. The cell is positioned in such a way that its axis of symmetry is vertically oriented. Due to buoyancy, the bubble rises to the top of the cell. The bubble is thus facing the inner part of the ceiling of the box. The cell is then submitted to vertical oscillations and the bubble behaviour is investigated. It is shown that the bubble splits into smaller parts if the acceleration of the oscillations exceeds a threshold. The experimental setup and the associated dimensionless groups are described in Section 2. Sections 3 and 4 are devoted to the experiments and subsequent results. A discussion is presented in Section 5. Finally, the findings are summarized in Section 6. 


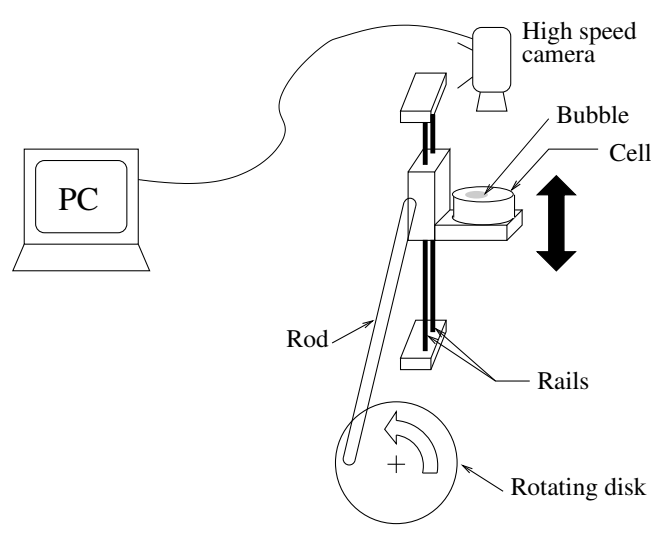

Fig. 1. Sketch of the experimental setup.

\section{Preliminary materials}

\subsection{Experimental setup}

The experimental setup is illustrated in Figure 1. It consists of a sealed cylindrical plexiglas cell of $8 \mathrm{~cm}$ diameter and $3 \mathrm{~cm}$ height. A $0.2 \mathrm{~cm}$ diameter hole was drilled through the side of the cylinder, providing an access for filling or emptying the cell. The hole is blocked with a nylon screw having the same length as the thickness of the wall. The cell is first fully filled with liquid and then, some of this liquid is removed with a graduated syringe to create a bubble inside the cell. The extracted volume is directly measured from the syringe graduations but is also weighted using an electronic balance having a precision of $0.1 \mathrm{~g}$. The bubble's initial volume is typically $V_{i}=4 \mathrm{ml}$. The cell is mounted onto a vertical rail with its axis of symmetry vertically oriented. The cell is mechanically vibrated by a scotch yoke mechanism (see Fig. 1). The amplitude $A$ of the oscillations can be varied from 0.5 to $3.0 \mathrm{~cm}$ by altering the distance between the rod pivot and the axis of the rotating disk. The rotating disk is driven by a SGMPH-08AA Fenwick servomotor which is capable of driving the system at an oscillation frequency $f$ up to $1600 \mathrm{rpm}$ with an accuracy of $2 \mathrm{rpm}$.

In the present experiments, distilled water, Rhodorsil silicon oil $47 \mathrm{~V} 10$, pure ethanol and mixtures of ethanol and water are used. The ethanol-water mixtures were made in order to investigate the influence of surface tension $\sigma$ on the bubble dynamics. Values of $\sigma$ were evaluated according to reference [20] by measuring the respective percentage in weight of each component. Surface tension values of these mixtures ranged from $72 \mathrm{dyn} / \mathrm{cm}$ for distilled water to $22 \mathrm{dyn} / \mathrm{cm}$ for pure ethanol. The influence of viscosity was investigated by comparing experiments using Rhodorsil silicon oil $47 \mathrm{~V} 10$ of viscosity $\mu=10 \mathrm{cP}$ and surface tension $\sigma=20.1 \mathrm{dyn} / \mathrm{cm}$ to those using a liquid of comparable surface tension, i.e. pure ethanol $(\mu=1.2 \mathrm{cP}$, $\sigma=22 \mathrm{dyn} / \mathrm{cm})$. All experiments are carried out at a room temperature of approximately $24^{\circ} \mathrm{C}$.

Experiments are carried out as follows. Due to buoyancy, the bubble rises to the top wall of the cell. Prior to each experiment the bubble is placed approximately

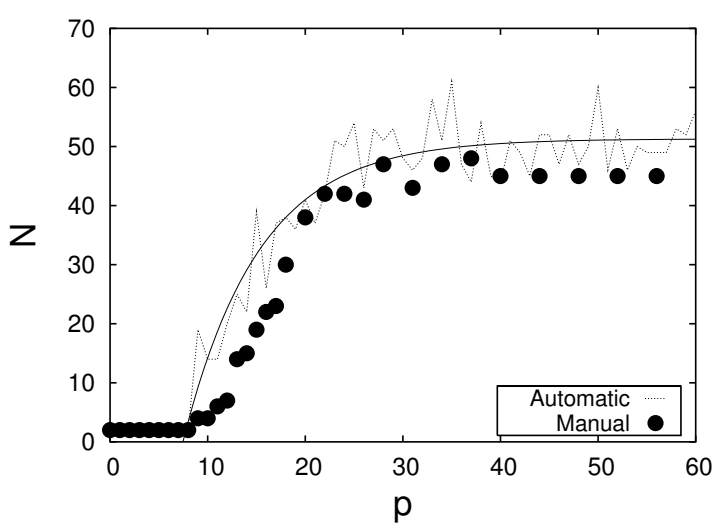

Fig. 2. Number of bubbles $N$ as a function of the number of periods $p$ accomplished by the cell. The dotted line and the data points represent the computer and manual readings, respectively. The solid line is a fit to the computational data using equation (5). Experimental parameters are: $A=0.5 \mathrm{~cm}$ and $f=600 \mathrm{rpm}, V_{i}=5.7 \mathrm{ml}$.

near the center of the upper surface and the latter is leveled with respect to the horizontal. The cell is then arbitrarily positioned at the top of the oscillating course before the system is set into motion. The motion of the bubble is captured from above with a Fastcam-Super 10k (Photron) high-speed video camera. The camera has a resolution of $512 \times 480$ pixels, yielding a spatial resolution of $1.7 \times 10^{-2} \mathrm{~cm}$. For all experiments, the camera shutter speed is set to $1 / 10000 \mathrm{~s}$ with a recording rate of 250 frames per second. The recorded images are then transferred to a computer for analysis.

\subsection{Image analysis}

An algorithm was developed in order to automate the determination of the number $N$ of bubbles present in the cell at a given time. In brief, on each frame of the sequence a threshold is used to highlight the bubble edges. Then, a particle analyzer algorithm is used to count the number of bubbles present in the cell as a function of the number of oscillations $p$ accomplished by the cell. In Figure 2, values of $N$ obtained with the program are compared with those manually counted. The results from the program show a systematic deviation of about $10 \%$ from the manual readings. This deviation is considered to remain within a reasonable interval of confidence [21].

\subsection{Dimensionless groups}

From parameters such as surface tension $\sigma$, amplitude of oscillations $A$, angular frequency $\omega=2 \pi f$ and density difference $\Delta \rho$ between air and liquid, a characteristic length $\ell$ can be defined as

$$
\ell=\sqrt{\frac{\sigma}{\Delta \rho A \omega^{2}}} .
$$


Moreover, considering the volume $V$ of the air bubble, its radius $r=(3 V / 4 \pi)^{1 / 3}$ can be compared to the magnitude of the oscillation amplitude $A$. This ratio $A / r$ evaluates the extent of squeezing of the bubble against the ceiling of the cell during an oscillation (see Sect. 3.1). The speed of this squeezing is characterized by the velocity $A \omega$. Dimensionless groups are thus defined as follows:

Reynolds number:

$$
R e=\frac{A \omega r \Delta \rho}{\mu}
$$

Froude number:

$$
F r=\frac{A \omega}{\sqrt{r g}}
$$

Bond number:

$$
B o=\frac{r}{\ell}=r \sqrt{\frac{\Delta \rho A \omega^{2}}{\sigma}} .
$$

In these groups, $g$ represents the gravitational acceleration. The Bond number indicates the relative importance of surface-tension-induced forces with respect to "effective" gravity.

\section{Experiments}

With the forcing oscillations, the bubble is observed to undergo a series of deformations which may lead to its splitting. In this section, the response of the bubble to the oscillations is described in the case of no splitting. The bubble dynamics with break-up will then be presented.

\subsection{Bubble dynamics without break-up}

Figures 3(a) and (b) show two sequences of top view images taken at $f=60 \mathrm{rpm}$ and $f=300 \mathrm{rpm}$, respectively. For both sequences, the amplitude is fixed at $A=0.5 \mathrm{~cm}$. The images are read from left to right, top to bottom and cover an oscillation period. At low frequency values (Fig. 3(a)) the bubble remains motionless during the whole oscillation period. Increasing the frequency (Fig. 3(b)) causes the bubble to be deformed. These deformations generate surface waves that are observed at the air/liquid interface. These waves appear to be closely shaped by the geometry of the bubble and share its axial symmetry. In Figure 3(b), one can see that the waves develop and travel from the periphery to the centre of the bubble. It also seems that they essentially arise when the bubble's diameter is decreased through deformations (see the last picture of the first line and the first two pictures of the second line in Fig. 3(b)).

During the oscillations the contact line between the top wall of the cell and the bubble is modified. Experimentally, we have observed that the displacement of the contact line induces the formation of a thin liquid film between the air and the Plexiglas wall. The process of the liquid-film formation, as sketched in Figure 4, is as follows. Before the

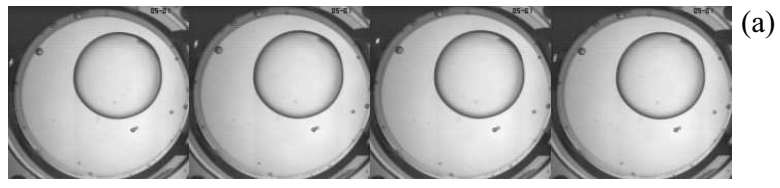

(a)

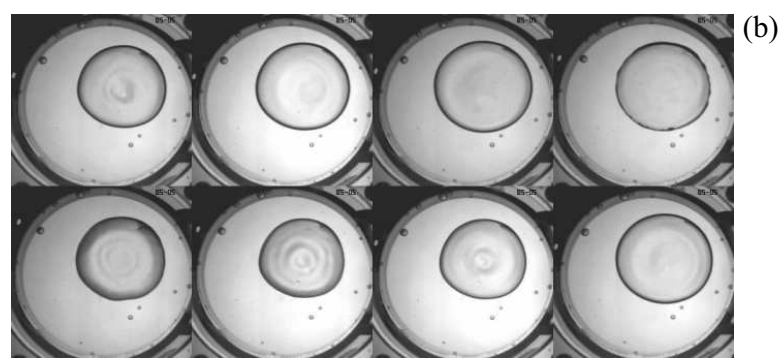

Fig. 3. Successive top views (from left to right, top to bottom) of the evolution of an air bubble in distilled water during an oscillation period. The experimental parameters are (a) $A=$ $0.5 \mathrm{~cm}$ and $f=60 \mathrm{rpm}$ (b) $A=0.5 \mathrm{~cm}$ and $f=300 \mathrm{rpm}$.

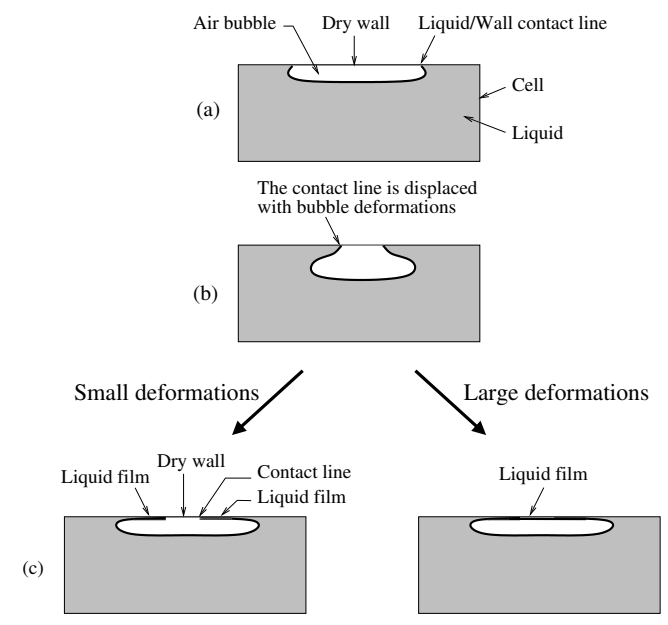

Fig. 4. Sketch of the wetting process of the top wall through the steps (a) at the start of the experiment, (b) deformation of the bubble during the oscillation period, (c) the formation of a liquid film when the bubble is squeezed against the top wall.

oscillations commence, the contact line between the liquid and the top dry wall is approximately located near the periphery of the bubble (Fig. 4(a)). As the setup oscillates, the bubble is deformed and the contact line between the liquid and the wall is displaced (Fig. 4(b)). This causes the wetting of the top surface. This wetting may be partial or total depending on the extent of bubble deformations. Once the bubble is squeezed again against the top wall, the liquid tends to withdraw from the surface. However, the oscillations are such that the liquid is never allowed to completely withdraw from the surface (Fig. 4(c)). Indeed, it is experimentally observed that a complete withdrawal of the liquid is only possible for frequencies substantially smaller than those considered here. For instance, by shaking the cell and then suddenly putting it to rest, we noted that the top surface becomes dry again after typically $\approx 1-5$ seconds. This time is much larger than the present oscillation periods. A liquid film is thus always present on 


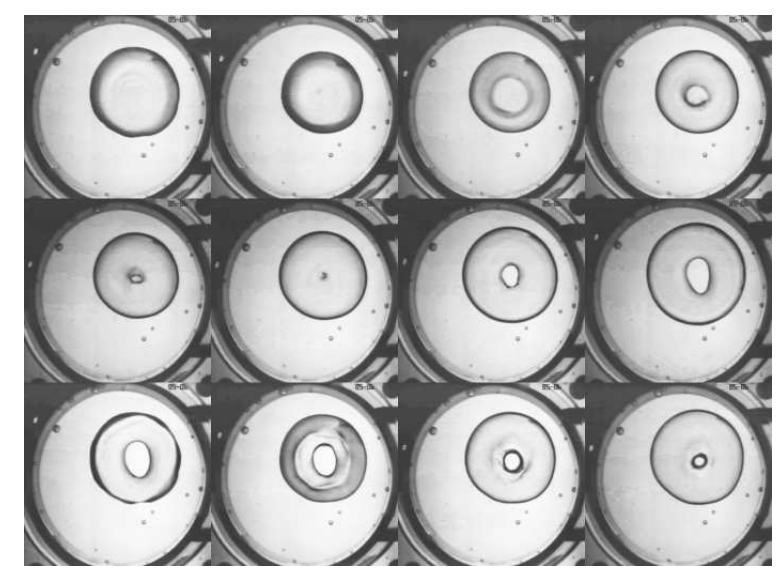

Fig. 5. Successive top views (from left to right, top to bottom) of an air bubble in distilled water during nearly two oscillation periods of the cell. The experimental parameters are $A=0.5 \mathrm{~cm}$ and $f=360 \mathrm{rpm}$.

the surface of the ceiling of the box for large deformations. It should be noted that the drainage mechanism of the liquid film between the air bubble and the cell wall is quite similar to what happens to the air film of a non-coalescing drop placed on a vibrating bath of the same liquid [22]. Couder et al. [22] have shown that in this configuration, the vibrations create a stable film between the drop and the liquid pool. Each descending motion helps in feeding the film with new fluid while the latter is drained in the ascending part.

By further increasing the frequency of the oscillations, the amplitude of the surface waves is amplified and the bubble exhibits a donut shape (Fig. 5). Once this donut is formed, it generally remains stable. However, in some cases, one can observe a temporary return of the bubble to its initial "lenticular" shape.

\subsection{Bubble dynamics with break-up}

For sufficiently large frequency values, surface waves are observed to invade the donut/torus along its centerline. These waves are superimposed on top of the deformations explained in the previous paragraph. Their appearance makes the bubble unstable and causes its split into several smaller bubbles. Figure 6 shows a typical sequence of images for bubble splitting. For further photographs and a video of the bubble splitting refer to reference [23] and [24], respectively. In Figure 6, surface waves appear (Fig. 6, first line) before the formation of a torus (Fig. 6, second line). Regularly spaced and almost symmetrical deformations are then observed along the centerline of the torus (Fig. 6, fourth line). Those deformations are characterized by large areas connected by narrow parts (nodes). The division of the bubble occurs when the cross-section of at least two nodes becomes so narrow that they eventually break up. Each newly created bubble follows the same process: they become toroidal, then unstable and eventually split into smaller parts. This process continues until the system reaches a final steady state where almost

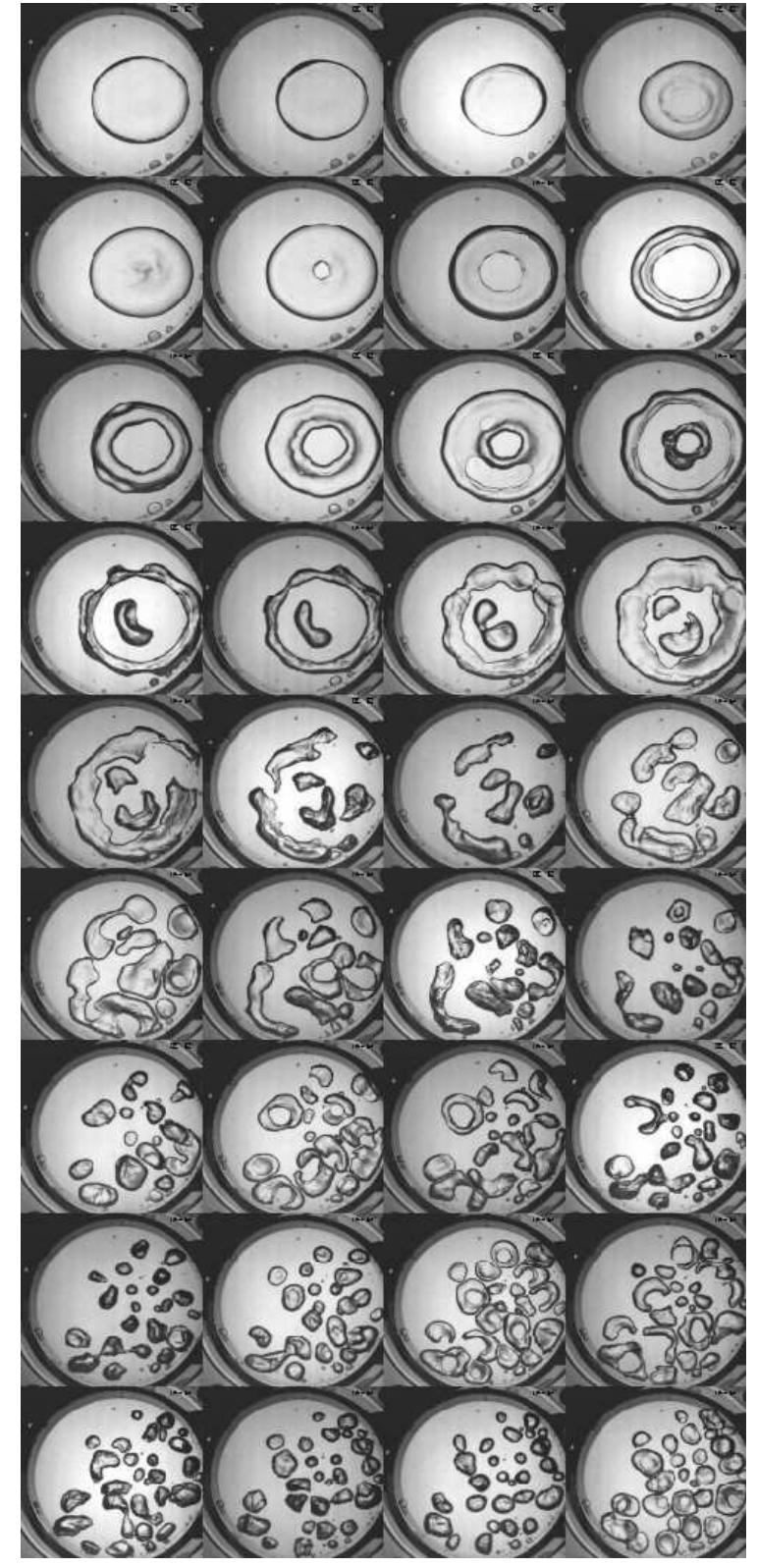

Fig. 6. Successive top views (from left to right, top to bottom) of an air bubble in distilled water during a few oscillations of the cell. Experimental parameters are $A=0.5 \mathrm{~cm}$ and $f=$ $720 \mathrm{rpm}$.

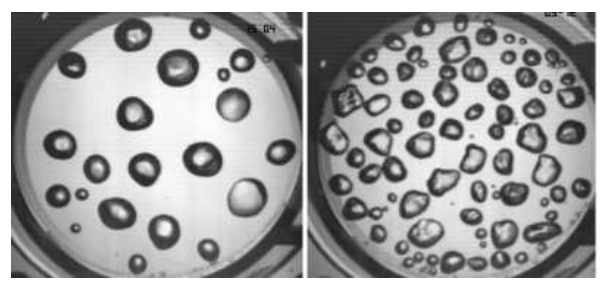

Fig. 7. After the final size is reached, bubbles tend to occupy the entire top surface of the cell and an almost regular spacing between them is observed. Experimental parameters are: (left) $A=1.5 \mathrm{~cm}$ and $f=240 \mathrm{rpm}$ and (right) $A=0.5 \mathrm{~cm}$ and $f=720 \mathrm{rpm}$. 


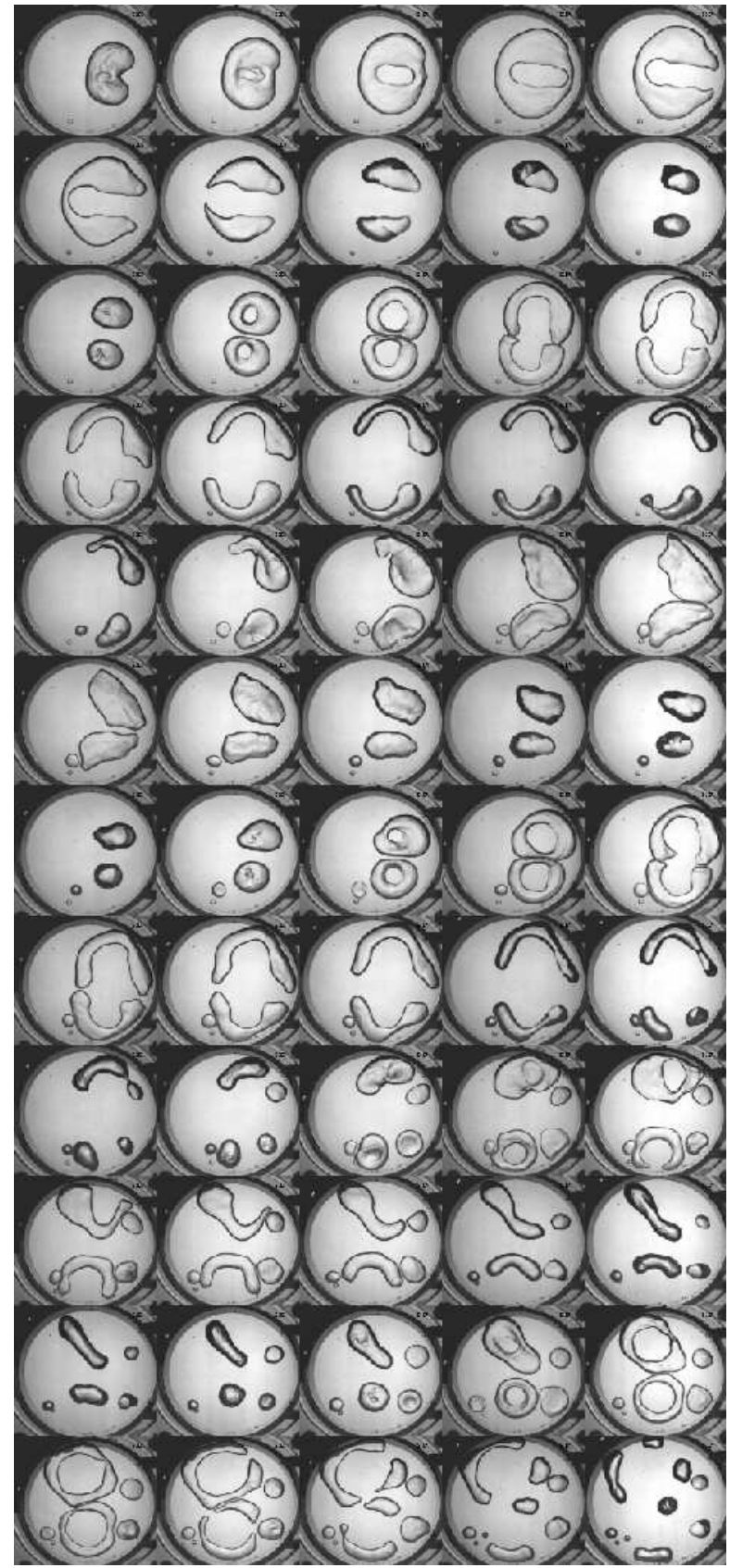

Fig. 8. Successive top views (from left to right, top to bottom) of an air bubble in distilled water during a few oscillations of the cell. The experimental parameters are $A=1.5 \mathrm{~cm}$ and $f=240 \mathrm{rpm}$.

no more division is observed. Thereafter, the number of bubbles present in the cell remains nearly constant.

Once the number of bubbles reaches a maximum $N_{f}$ the bubbles start to occupy the entire top surface of the cell and an almost regular spacing between them is observed (Fig. 7). One should note that, in most cases, occasional collisions between the bubbles do not lead to any coalescence.

Figure 8 presents another interesting example of bubble division process. In this case, a vertical plane of sym-

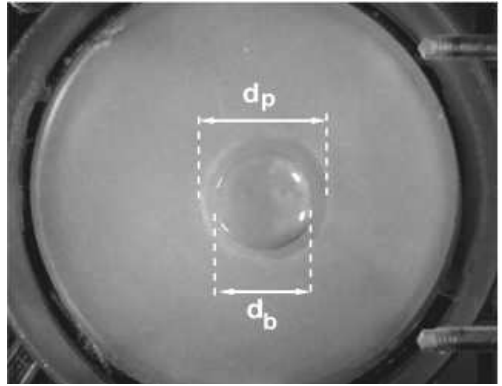

Fig. 9. Top view of a cell containing Kalliroscope particles to help visualize the flow. The bubble size $d_{b}$ and the size of perturbated region $d_{p}$ are illustrated.

metry along the diameter of the cell is observed during the experiment. This particular symmetry mostly occurs for relatively large amplitude $(A \gtrsim 1.5 \mathrm{~cm})$ and low frequency $(f \lesssim 4 \mathrm{~Hz})$ values. It is further associated with relatively small $N_{f}(\lesssim 20)$ values.

Observations of the bubble from the side have also been performed by placing the video camera perpendicular to the oscillation plane. Within the range of $f$ and $A$ values tested here, side observations confirmed that the bubbles never touched the bottom wall of the cell. One should also note that the present results are reproducible and robust.

\subsection{Flow motion in the cell}

As the bubble is moving relatively to its ambient liquid, flow motion might be induced inside the cell. In order to visualize the liquid flow, some Kalliroscope particles were added to the liquid. The experiments show that as long as the bubble of diameter $d_{b}$ does not split, the fluid motion is confined to a circular region of diameter $d_{p}$ surrounding the bubble (Fig. 9). Fluid motion is thus induced only in the close neighborhood of the bubble. Quantitative measurements showed that the ratio $d_{p} / d_{b}$ ranges from 1 to 2.2 before bubble division. However, once the bubble is broken, the flow perturbation is observed to propagate everywhere in the cell. This is due to the collective effect of the large number of small bubbles in motion.

\section{Results}

In order to characterize this system, our experimental approach has focused on: i) defining the onset for bubble division and then ii) characterizing the bubble response to oscillations above this onset.

The onset for bubble division has been studied by fixing the oscillation amplitude $A$ and gradually increasing its frequency $f$ until a division was observed. The rotation speed was increased in steps of $10 \mathrm{rpm}$. Figure 10(a) shows the resulting phase diagram of the bubble division as a function of both angular speed $\omega$ and amplitude $A$ for different initial volume values $V_{i}$ of an air bubble in distilled water. Data points correspond to the onset of bubble division. Above these points, an initial bubble divides into 

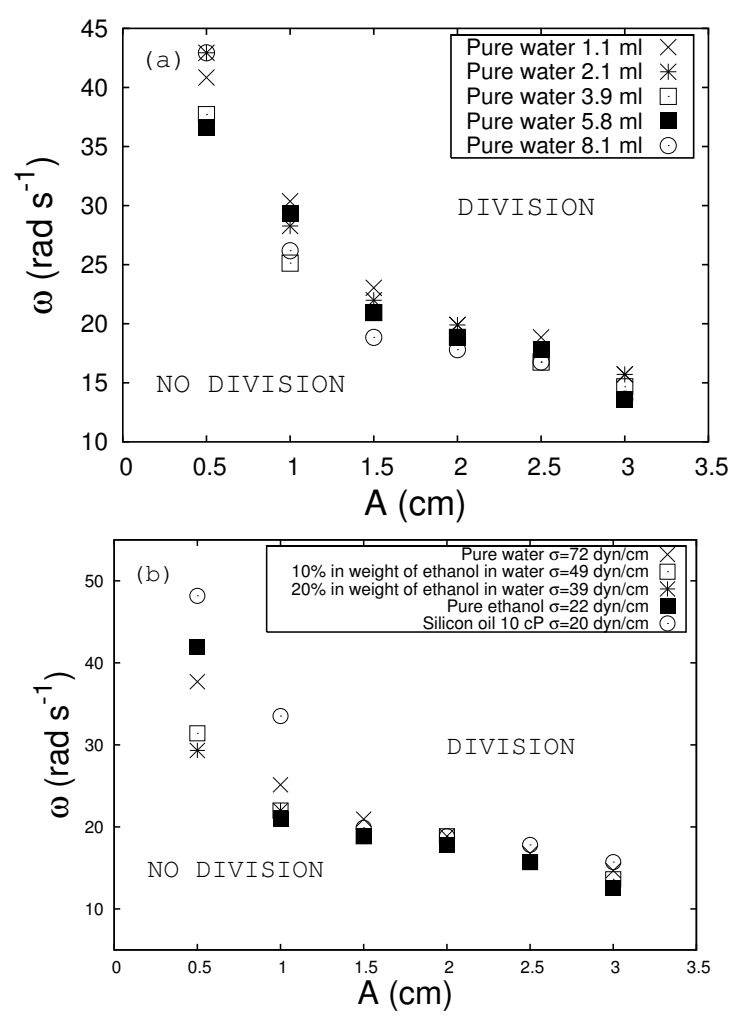

Fig. 10. Phase diagram of bubble split for (a) different initial volume $V_{i}$ of an air bubble in distilled water, (b) different surface tensions $\sigma$ and a volume $V_{i}=4 \mathrm{ml}$.

several smaller parts; below them, no division is observed. Figure 10(a) appears to reveal that a variation of $V_{i}$ has a negligible effect on the onset of bubble division within the range of $V_{i}$ values that were tested $\left(1<V_{i}<8 \mathrm{ml}\right)$. Furthermore, a variation in $V_{i}$ implies a modification of the aspect ratio between the bubble volume and that of the cell. Therefore, the above results and previous observations of the fluid flow in the cell (Sect. 3.3, Fig. 9) suggest that, for the range of $V_{i}$ tested here, the size of the cell does not seem to influence the physical mechanism of bubble division.

Fitting the data sets of Figure 10(a) shows that the onset of bubble division is governed by the following relationship:

$$
\omega^{2}=c A^{-1},
$$

where $682 \pm 9<c<840 \pm 19 \mathrm{~cm} \mathrm{~s}^{-2}$ depends on $V_{i}$. Since $c$ slightly depends on $V_{i}$ and from equation (4) it follows that the bubble splitting is primarily governed by the acceleration $\Gamma=A \omega^{2}$ of the cell. Figure 10(b) emphasizes the influence of the surface tension $\sigma$ on the onset of bubble splitting. This figure suggests that changing $\sigma$ has only a small effect on the onset of bubble division. In this figure, data corresponding to different viscosities are also illustrated. As the surface tension of silicon oil is close to that of pure ethanol, the influence of viscosity can be evaluated by comparing their respective experimental data. In Figure 10(b) data corresponding to the silicon oil $(\mu=10 \mathrm{cP})$ are close to those of ethanol $(\mu=1.2 \mathrm{cP})$.
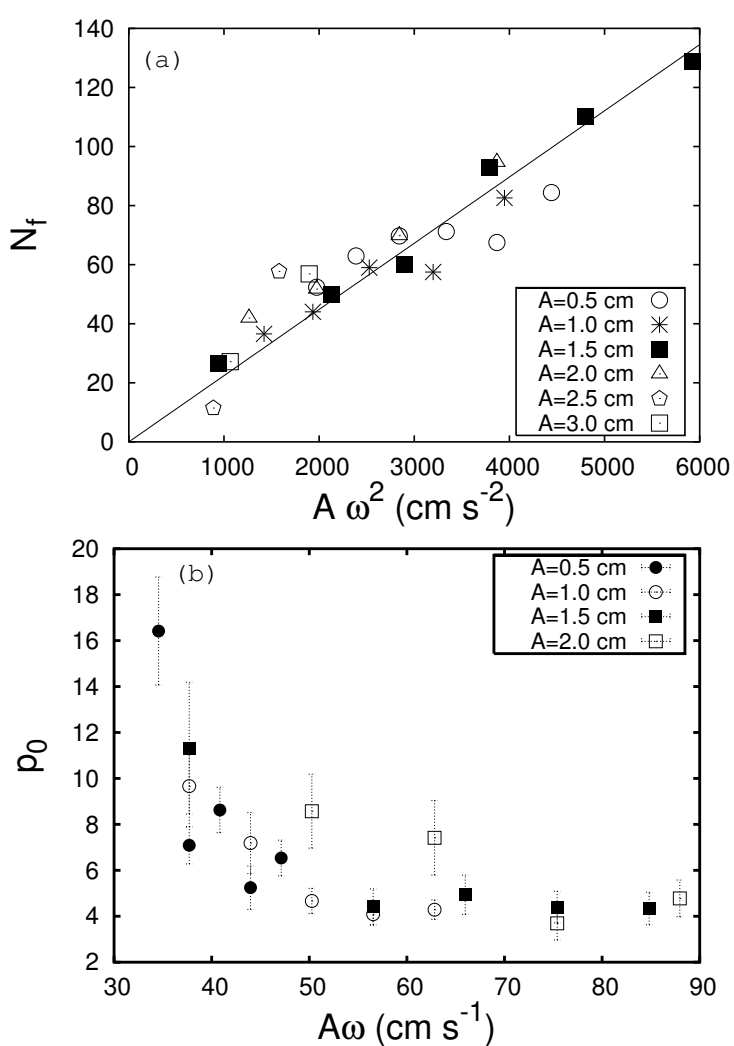

Fig. 11. (a) The asymptotic number of bubbles $N_{f}$ in the cell as a function of the acceleration $\Gamma=A \omega^{2}$. (b) The rate of bubble division $p_{0}$ as a function of velocity $A \omega$ for different amplitudes. The bubble initial volume is $V_{i}=5.7 \mathrm{ml}$.

It thus appears that the onset of bubble splitting is not modified by a change in liquid viscosity, within the range of $\mu$ values tested here.

Once the acceleration threshold is exceeded, the number $N$ of bubbles in the cell evolves as a function of the number $p$ of oscillations accomplished by the cell. A typical curve is presented in Figure 2. One can observe a growth of $N$ with $p$ until a final steady state is reached. The growth rate of $N$ was studied by assuming the following law:

$$
N(p)=N_{f}\left(1-\exp \left(-p / p_{0}\right)\right)-N_{0}
$$

where $N_{f}$ represents the asymptotic number of bubbles in the cell, $N_{0}$ is the initial number of bubbles in the cell $\left(N_{0}=1\right)$ and $p_{0}$ is the rate of bubble division. The relevancy of this empirical law is illustrated in Figure 2. Both $N_{f}$ and $p_{0}$ characterize the temporal evolution of $N$ in terms of the number of oscillations. We have observed that $N_{f}$ grows with both $A$ and $\omega$, while $p_{0}$ seems to decrease with $\omega$. Individual data sets appear to collapse on a single curve when $N_{f}$ and $p_{0}$ are plotted as functions of $A \omega^{2}$ (Fig. 11(a)) and $A \omega$ (Fig. 11(b)), respectively. A leastsquares fit to the data of Figure 11(a) shows that $N_{f}$ is simply proportional to the acceleration $A \omega^{2}$ of the cell. A fit to the data of Figure 11(b) was not performed as relatively large scatter in this data was observed. 


\section{Discussion}

The physical quantities which might be important in the stability of a bubble are its volume, the surface tension and the basic flow [25]. In this section, the roles played by these quantities in the mechanism of bubble division are described.

\subsection{The process of a bubble division}

It has been observed that the bubble division is composed of three main stages. A discussion of each of these stages in their order of appearance during the bubble splitting process is presented below.

\subsubsection{First stage: the formation of a torus}

During the oscillations, bubbles larger than the characteristic length $\ell$ are flattened by apparent gravity. For sufficiently large $\Gamma$, the oscillating motion of the cell leads to surface waves at the liquid/air interface. The wave amplitude is observed to amplify with increasing frequencies. These waves are also observed to develop and travel from the periphery to the centre of the bubble. Furthermore, they are axi-symmetrical and close up at the center of the bubble where they reach maximum amplitudes. It is at this position that both upper and bottom sides of the bubble merge, leading to the formation of a toroidal (donut) bubble. In some marginal cases a bubble split without the prior formation of a torus was observed. Here, the study is restrained to the case where torus formation precedes the splitting of the bubble. Bubbles smaller than $\ell$ are less deformed and may then remain stable. The correlation bewteen bubble split and characteristic length $\ell$ will be emphasized in Section 5.2. It should be noted that donut shape drops have also been reported in the case of Leidenfrost drops [26]. Biance et al. [26] have shown that drops larger than a critical radius may take the donut shape due to the appearance of rising bubbles. This critical size scales as the capillary length, similarly to our characteristic length $\ell$.

\subsubsection{Second stage: a reduced gravitational acceleration}

In the reference frame of the cell, the resultant force $F_{t o t}$ applied on the bubble reads

$$
F_{\text {tot }}=F_{\text {buoy }}+F_{a c c} \simeq-\Delta \rho V\left(g-A \omega^{2} \cos (\omega t)\right),
$$

where $F_{\text {buoy }}$ is the buoyant force, $F_{a c c}$ is the force due to the acceleration and $t$ is the time. Here, we have $\Delta \rho \approx \rho_{f}$.

Forces arising from the acceleration of the cell and from buoyancy are parallel and share the same direction in the lower part of the oscillating course, while they are in opposite directions in the upper part. Hence, the resultant force $F_{\text {tot }}$ on the bubble decreases in the upper part of the oscillating motion and attains a minimum value at

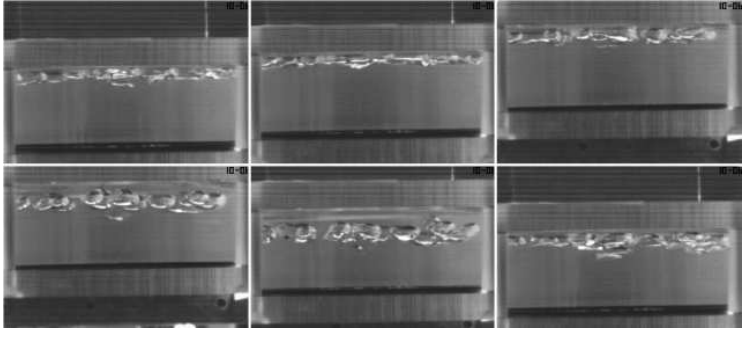

Fig. 12. Successive side views of the cell. Pictures, read from left to right and from top to bottom, are taken for oscillation parameters $A=1 \mathrm{~cm}$ and $f=360 \mathrm{rpm}$. On the first line the pictures show the cell heading to its top position, while on the second line the cell is moving to the bottom position.

the top position. According to equation (4), the onset for bubble division occurs when the bubble experiences a reduced gravitational acceleration $g^{\prime}=g-A \omega^{2}$ with $(0.14 \pm 0.01) g \lesssim g^{\prime} \lesssim(0.30 \pm 0.02) g$. This implies that the resultant force on the bubble is small at the onset of bubble division. Therefore, surface tension becomes the relevant force in shaping the bubble. Thus, the equilibrium shape of a bubble (torus) is expected to be spherical (annular) in order to minimize its surface for a given volume [27]. This reduces the "contact" area between the bubble and the upper wall and we consider it as a partial detachment of the bubble.

With respect to the above physical arguments, for values of $\Gamma$ larger than $g$, a complete detachment of the bubble from the top wall is expected. This was experimentally verified by side observations. Figure 12 presents successive images of an already divided bubble during one period of oscillation for $\Gamma=1421 \mathrm{~cm} \mathrm{~s}^{-2}$. The top line of this figure shows the ascending cell, while the bottom line corresponds to the descending motion. The bubbles are observed to stick on the top wall in the half lower part of the oscillating course and detach from it in the upper part. This is in agreement with previous statements. It is interesting to note that in Figure 12 all bubbles reach a nearly spherical shape at the top position of the oscillations (bottom left picture), i.e. when the bubbles are in a state of reduced gravity.

In order to evaluate the influence of viscosity $\mu$ on the vertical motion of the bubble, the Reynolds number has been calculated. It is found that the order of magnitude of $R e$ lies between $10^{2}$ and $10^{3}$ at the onset. This suggests that the influence of fluid viscosity is negligible compared to that of fluid inertia. The values of $R e$ are, however, observed to fall down to $60 \lesssim R e \lesssim 2500$ for experiments above the threshold when the bubbles are at their final stable size. A decrease in Re is natural since with increasing velocity the size of the bubbles decreases, meaning that the effect of viscosity is likely to become important. However, the drop in Re by up to two orders of magnitude might give rise to a change in the system response to the oscillations. This is addressed in Section 5.2 where it is shown that despite this sharp fall, the influence of viscosity on the system seems to be limited. 


\subsubsection{Third stage: bubble division}

Cheng and Holtham [19] observed a circular motion of monazite particles attached to a vertically oscillating bubble. They associated the particle motion solely to centrifugal forces. The authors suggest that the observed circular motion of the particles should, to some extent, also be related to the surrouding flow motion. In the present experiment the appearance of the torus is considered to induce recirculation in the surrounding fluid. Meanwhile, surface tension tends to deform/pinch the torus along its axis ${ }^{1}$. The fluid motion around the torus seems to amplify these deformations, leading to the division of the bubble.

\subsection{Size scaling of the bubbles with the oscillation parameters}

The ratio between inertia and gravity has been investigated using the Froude number (Eq. (2)). Figure 13(a) shows the Froude number as a function of the aspect ratio $A / r$ for data obtained at the onset of bubble division. As previously mentioned, the parameter $r$ represents the radius $r=(3 V / 4 \pi)^{1 / 3}$ of a bubble of volume $V$. It is seen that all values collapse on one single curve regardless of the initial bubble size, the fluid viscosity or the surface tension. Moreover, $\mathrm{Fr}$ is observed to increase from 0.5 to 1.9 with increasing $A / r$ values. The solid line in this figure corresponds to a power law fit giving $F r=0.84(A / r)^{0.48}$. This yields to the relation $A \omega^{2} / g \approx 0.7$. This accelerationto-gravity ratio at the onset is in agreement with observations of Section 4 (Eq. (4)).

As bubble division implies smaller $r$ values, this relationship between gravity and cell acceleration is expected to break down. Indeed, for a fixed value of $A$, the aspect ratio $A / r$ increases with decreasing bubble size $r$. However, decreasing $r$ implies a growth in capillary pressure inside the bubble. The latter is inversely proportional to the curvature radius (see, e.g., $[28,29]$ ). Therefore, a very small bubble is unlikely to break-up [30,31]. The growing importance of surface tension as the bubble size decreases also implies the non-dependence between $V_{i}$ and the bubble division threshold (Figs. 10(a) and 13(a)) should only be true above a critical initial size $V_{i c}$. For initial volumes below $V_{i c}$ no amount of vibration would be sufficient to overcome capillary forces. Unfortunately, the range of $A / r$ provided by the present setup did not allow an experimental determination of $V_{i c}$.

Competition between "effective" gravity and surfacetension-induced forces is characterized by the Bond number (Eq. (3)). Figure 13(b) shows the Bond number as a function of the aspect ratio $A / r$. Data obtained both at and above the onset for bubble division are illustrated. In

\footnotetext{
${ }^{1}$ Note that the characteristic wavelengths observed in the experiments are of the order of $\mathrm{cm}$. This is of the same order of magnitude as for wavelengths derived by both Rayleigh-Taylor or Rayleigh-Plateau calculations. Therefore, at the present state of art we cannot give with conviction the exact nature of the observed pinching.
}

(a)

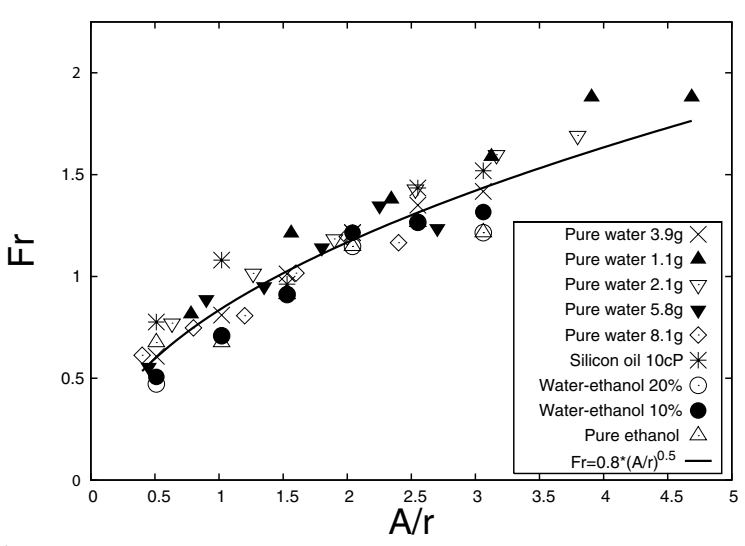

(b)

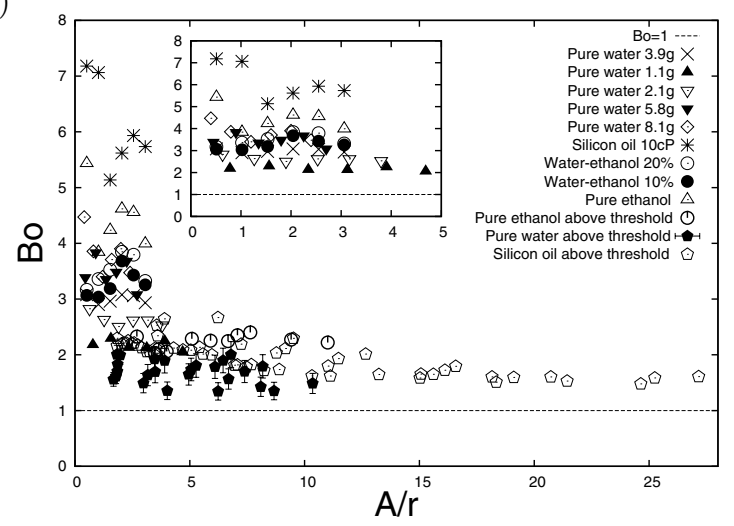

Fig. 13. Ratio $A / r$ between the amplitude $A$ of oscillations and the bubble radius $r$ as a function of (a) Froude number $F r=A \omega / \sqrt{r g}$, (b) Bond number $B o=r \sqrt{\Delta \rho A \omega^{2} / \sigma}$. Please note that error bars are related to data obtained from image analysis, while other bubbles were manually counted.

this figure data with error bars were obtained by image analysis algorithm, while others were evaluated manually. The small graph is a rescaled plot of data obtained at the onset. This graph will be discussed first.

In the enclosed graph of Figure 13(b), the Bond number exhibits values ranging as $2 \lesssim B o \lesssim 8$. For individual experimental data sets the Bond number seems constant. It also appears that Bo slightly increases with fluid viscosity, as revealed by experiments carried out with the same initial bubble size and comparable surface tensions (pure ethanol and $10 \mathrm{cP}$ silicon oil). Although the Reynolds number was found of the order $10^{2} \lesssim R e \lesssim 10^{3}$ for experiments at the onset, the above result might suggest that the fluid viscosity has some influence on the threshold for bubble division.

It should be noted that, as a function of $A / r$ ratio, Bond and Froude numbers lead to a three-dimensional phase diagram for bubble break-up threshold.

Above the acceleration threshold (Fig. 13(b), main plot) the dependence between the Bond number and the fluid viscosity is no more observed. Indeed, above the onset, the final and stable size of bubbles appears to become small enough for the surface tension to become the relevant force where the Bond number tends to unity. The 
pressure inside the bubbles increases as their size becomes smaller. All bubbles become then harder to deform. This might explain the reason why at the onset a dispersion and viscosity dependence of $B o$ was previously observed. Indeed, the relevancy of surface tension is less important for large bubbles for which deformations and flattening due to wall effects can occur.

It should be noted that the square-root dependence of $\ell_{c}$ on $\sigma$ could be responsible for the small influence of the surface tension value on the onset for bubble division. The slight dependence on the onset upon surface tension might also be seen as resulting from the followings. A decrease of the surface tension leads to a more easily deformable bubble. This should thus provide an "easier" split of the bubble as deformations are needed. On the other hand, capillary break-ups are enhanced by an increase of surface tension. A decrease of $\sigma$ is thus resulting in more difficulty in splitting the bubble. The variation of surface tension appears then to have antagonist effects on the break-up which probably explains the small variations of the breakup onset with $\sigma$.

\section{Summary}

Experiments have been performed on a bubble subject to vertical vibrations within a sealed cylindrical cell. The bubble is observed to split into smaller parts when the acceleration of the cell $\Gamma$ reaches a threshold. Above this threshold, the number of bubbles present in the cell grows until a final steady state is reached. After this, the number of bubbles remains constant. This final number of bubbles is found to depend on the acceleration of the cell. Dimension analysis shows that the threshold for bubble division as well as the final steady state of the system are characterized by a Bond number close to unity.

H.C. is a FNRS (Brussels, Belgium) researcher. This work was financially supported by CNES (France) as well as by ESA through the project AO-99-108.

\section{References}

1. J.O. Hinze, Am. Inst. Chem. Eng. J. 1, 289 (1955).

2. D. Weaire, S. Hutzler, The Physics of Foams (Clarendon Press, Oxford, 1999).

3. D. Lohse, Phys. Today 56, 36 (2003).

4. P.P. Wegener, J.-Y. Parlange, Annu. Rev. Fluid Mech. 5, 79 (1973).
5. J. Magnaudet, I. Eames, Annu. Rev. Fluid Mech. 32, 659 (2000).

6. J. Rensen, D. Bosman, J. Magnaudet, C.-D. Ohl, A. Prosperetti, R. Tögel, M. Versluis, D. Lohse, Phys. Rev. Lett. 86, 4819 (2001).

7. M. Faraday, Philos. Trans. R. Soc. London 52, 299 (1831).

8. S. Douady, J. Fluid Mech. 221, 383 (1990).

9. S. Fauve, K. Kumar, C. Laroche, D. Beyssens, Y. Garrabos, Phys. Rev. Lett. 68, 3160 (1992).

10. H. Rodot, C. Bisch, A. Lasek, Acta Astron. 6, 1092 (1979).

11. A.J. James, B. Vukasinovic, M.K. Smith, A. Glezer, J. Fluid Mech. 476, 1 (2003).

12. A.J. James, M.K. Smith, A. Glezer, J. Fluid Mech. 476 , 29 (2003).

13. E.D. Wilkes, O.A. Basaran, J. Colloid Interface Sci. 242, 180 (2001).

14. E.D. Wilkes, O.A. Basaran, Phys. Fluids 9, 1512 (1997).

15. J. Ellenberger, J.M. van Baten, R. Krishna, Catal. Today 79-80, 181 (2003).

16. R.I. Nigmatulin, I.Sh. Akhatov, N.K. Vakhitova, R.T. Lahey, J. Fluid Mech. 414, 47 (2000).

17. M. Kawaji, N. Ichikawa, A. Kariyasaki, A.B. Tryggvason, Large bubble motion in a fluid cell under microgravity: ISCAP experiments on the effects of g-jitter and forced vibration, IAF Paper 99-J308 (1999) (available at http://www.space.gc.ca/asc/pdf/2000_kawaji_1.PDF).

18. M. Ishikawa, T. Nakamura, S. Yoda, H. Samejima, T. Goshozono, Microgravity Sci. Technol. 7, 164 (1994) (available at http://www.space.gc.ca/asc/pdf/ 2000_FRIESEN.PDF).

19. T.W. Cheng, P.N. Holtham, Miner. Eng. 8, 883 (1995).

20. T. Reis, Introduction à la chimie-physique des surfaces (Editions Dunod, Paris, 1952).

21. H. Caps, H. Decauwer, M.-L. Chevalier, G. Soyez, M. Ausloos, N. Vandewalle, Eur. Phys. J. B 33, 115 (2003).

22. Y. Couder, E. Fort, C.H. Gautier, A. Boudaoud, Phys. Rev. Lett. 94, 177801 (2005).

23. F. Zouestiagh, M. Legendre, N. Vandewalle, H. Caps, Phys. Fluids 16, S7 (2004).

24. http : // pof .aip.org/pof/gallery/2004-Zoueshtiagh. jsp.

25. T.J. Pedley, J. Fluid Mech. 32, 97 (1968).

26. A.L. Biance, C. Clanet, D. Quéré, Phys. Fluids 15, 1632 (2003).

27. H.K. Moffatt, Microhydrodynamics, lecture notes, Vol. 1 (Ed. Ecole Polytechnique, Palaiseau, 1996).

28. R.F. Probstein, Physicochemical Hydrodynamics, an Introduction (Butterworth, Boston, 1989).

29. A.W. Adamson, Physical Chemistry of Surfaces, fifth ed. (John Wiley \& Sons Inc., NY, USA, 1990).

30. T. Wang, J. Wang, Y. Jin, Chem. Eng. Sci. 58, 4629 (2003).

31. V.G. Levich, Physicochemical Hydrodynamics (PrenticeHall, Inc., Englewood Cliffs, NJ, 1962). 